\title{
Knowledge, Attitudes and Practices of Pediatricians about Effects of Pediatric Drugs on Oral Health: A Survey Study
}

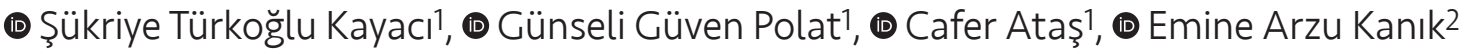

1University of Health Sciences Turkey, Department of Pediatric Dentistry, İstanbul, Turkey

2 Mersin University Faculty of Medicine, Department of Biostatistics, Mersin, Turkey

\begin{abstract}
Aim: Pediatricians may be able to play an important and effective role in providing forward guidance on children's oral health. When they make the right recommendations, the erosive, cariogenic and staining effects of medications are predicted to reduce. The objective of this research is to assess the knowledge, attitude and practices of pediatricians concerning the effects of medication use on oral health.

Materials and Methods: A cross-sectional, descriptive survey study was conducted on pediatricians in İstanbul, Turkey. Data were collected by distributing a questionnaire to pediatricians working in hospitals and private clinics. Differences between the proportions of categories were assessed using the chi-square tests. For the differences between two ordinal variables, rank sums nonparametric test Mann-Whitney $\mathrm{U}$ test was used for quantitative analysis.

Results: $54.3 \%$ of the responders informed patients about oral health after prescribing drugs. Only $27 \%$ of the pediatricians felt knowledgeable about informing parents about oral care after drug use. Only $17.7 \%$ of them agreed with the statement that their training about oral health during medical school/pediatrics is adequate.

Conclusion: There is a need to organize the curriculum relating to oral health in pediatrics education and to increase the level of knowledge of pediatricians through educational programmes.
\end{abstract}

Keywords: Oral health, pediatrician, dental caries, pediatric drug, tooth staining

\section{Introduction}

Liquid oral medicines are extensively recommended for children. Acids are added to medications as a buffering agent to maintain chemical stability to maintain tonicity and physiological compliance and also sugars are added to enhance the taste, consistency and compatibility of the medication $(1,2)$. The high frequency of intake, low $\mathrm{pH}$, high titratable acidity, high viscosity, bedtime consumption, salivary flow reduction and high sugar content affects the cariogenic and erosive potential of these medications $(3,4)$.
Iron supplements may lead to caries and dental erosion in addition to one of its main problems being tooth staining. Tooth staining is a major cause of concern among parents and can adversely affect the social interactions of preschool children (5). The ionic form of ferric sulfide has been suspected as it may interact with gingival cervical fluid and bacterial hydrogen sulfide to produce iron stains (6). Additionally, powdered or aerosol versions of inhaled medications are acidic and have erosive potential especially when used on a regular basis and over long period of time (7).

\section{Address for Correspondence}

Şükriye Türkoğlu Kayacı MD, University of Health Sciences Turkey, Department of Pediatric Dentistry, Istanbul, Turkey Phone: +90 5555109396 E-mail: sukriyeturkoglu1@gmail.com ORCID: orcid.org/0000-0002-1477-5283 Received: 05.11 .2019 Accepted: 15.01 .2020

${ }^{\circ}$ Copyright 2020 by Ege University Faculty of Medicine, Department of Pediatrics and Ege Children's Foundation The Journal of Pediatric Research, published by Galenos Publishing House. 
Many recommendations have been made for minimizing tooth damage caused by medications. The patient should be advised to rinse the mouth immediately after taking the medicine (8). Immediate toothbrushing is not recommended because of the increased risk of abrasive wear on the softened/eroded surface and so it should be delayed for at least 20 min after an erosive attack and possibly up to 60 $\min (9)$.

Parents infrequently bring their children to visit a dentist to check dental diseases. Pediatricians are the main contributors of premier healthcare for children due to their frequent communication with families for check-up visits in the early years of a child's life. They can play an important and effective role in providing forward guidance on children's oral health (10). The American Academy of Pediatrics (AAP) places emphasis on the substantial role of pediatricians in oral health (10). When the right recommendations are made, the erosive, cariogenic and staining effects of medications are predicted to reduce.

Although there are some published articles researching the knowledge and attitudes of pediatricians towards pediatric medicines and their cariogenic and erosive potentials, there is no published data on pediatrists' knowledge and attitudes on suspension or inhaler form medications' effects and no data about their knowledge and attitudes of iron supplements' staining effects.

The objective of this research is to assess the knowledge, attitude and practices of pediatricians on the effects of medication use on oral health.

\section{Materials and Methods}

Ethics approval was obtained from the institutional ethics and research committee of the University of Health Sciences (12/18) (Approval number:18/103). Informed consent was taken from the people who participated in study.
A cross-sectional, descriptive survey study was conducted on pediatricians in Istanbul, Turkey. In order to estimate their knowledge, attitudes and practices on tooth decay, dental erosion and tooth staining relating to pediatric medications, a survey was carried out. Certain tools utilized in previous studies were adapted to this survey. We utilized material from previous studies that investigated the relationship between dental caries and dental erosion $(3-5,7)$, and also tooth staining (6). A draft questionnaire was prepared and this was evaluated by one pediatrician, one pediatric dentist and one biostatistics specialist.

The questionnaire included items concerning demographics, most frequently prescribed medicaments and also knowledge, attitudes and practices about the effects of medication use on oral health. Pediatricians were asked about the erosive, dental caries and tooth staining effects of suspensions, inhalers and iron preparations. In addition; pediatricians were asked about their oral health recommendations after prescribing medications. In relation to some items on the questionnaire, there was the choice of marking a single answer, for instance 'true, false or not sure' or the options 'agree, disagree or not sure' for other items. There was also an open-ended question.

Random cluster sampling was used (Table I). An estimated 300 pediatricians were included in this study.

Data were collected by distributing the questionnaire to those pediatricians working in hospitals and private clinics. Initially, an informed consent form that explained the objectives of the study and ensured data confidentiality was completed. Following this, the surveys were distributed and gathered in on the same day (via the face-to-face method). Data were collected between January $1^{\text {st }}$ and March 29, 2019.

www.e-picos.com, New York was utilized for the statistical analysis of the collected data. Chi-square tests were used to examine the differences between proportions of categories. The number of daily patients and years

Table I. Random cluster sampling methodology of the work

\begin{tabular}{|l|l|l|l|l|l|l|l|}
\hline & \multicolumn{2}{|l|}{$\begin{array}{l}\text { Real } \\
\text { population } \\
\text { Size (private } \\
\text { hospital) }\end{array}$} & $\begin{array}{l}\text { Real } \\
\text { population } \\
\text { size (public } \\
\text { hospital) }\end{array}$ & $\begin{array}{l}\text { Estimated } \\
\text { population } \\
\text { size (private } \\
\text { hospital) }\end{array}$ & $\begin{array}{l}\text { Estimated } \\
\text { population } \\
\text { size (public } \\
\text { hospital) }\end{array}$ & $\begin{array}{l}\text { Actual } \\
\text { population } \\
\text { size (private } \\
\text { hospital) }\end{array}$ & $\begin{array}{l}\text { Actual } \\
\text { population } \\
\text { size (public } \\
\text { hospital) }\end{array}$ \\
\hline Research assistant & 481 & 105 & 71 & 79 \\
\hline Specialist & 466 & 358 & 103 & 4 & 3 & & 7 \\
\hline Associate professor & 16 & 14 & 4 & 2 & & 300 \\
\hline Professor & 20 & 8 & 4 & 300 & & \\
\hline Total & 1363 & & & & \\
\hline
\end{tabular}


of practice were divided into 4 groups using median and quartiles (25-75). For the differences between two ordinal variables, rank sums non-parametric test Mann-Whitney $U$ tests were used for quantitative analysis. P-value of less than 0.05 was accepted as a statistically significant difference. Qualitative data analysis was used for open-ended questions.

\section{Results}

\section{Responder Characteristics}

The 28 questions included on the questionnaire were answered by 300 pediatricians. The questionnaires were carried out by a hand delivery system to increase the response rate and reliability.

All responders were from İstanbul, TURKEY. The median age was 38 [IQR (30.46)] and 60.7\% were female. The demographic characteristics of the responders are shown in Table II.

\section{Knowledge}

$58 \%$ of pediatricians agreed that dental caries and $49.3 \%$ that dental erosion due to the use of suspension form drugs (antibiotics, analgesics) is expected. $44.3 \%$ of pediatricians agreed that dental caries and $39.7 \%$ that dental erosion due to the use of iron preparations is expected. Most (90\%) pediatricians agreed that tooth staining due to iron preparations is expected. $41 \%$ of pediatricians agreed that dental caries and $55.3 \%$ that dental erosion due to the use of inhaler drugs is expected. Also $76.3 \%$ of them agreed that taking medication before going to bed at night may increase the risk of dental caries.

\section{Attitudes and Practices}

The majority of pediatricians (47\%) agreed that the acidity of the drugs that they prescribe is important for them. Most of them (62.7\%) agreed that the sugar content of the drugs that they prescribe is important for them. $70.3 \%$ of pediatricians recommended that their patients rinse their mouth after using suspension/inhaler drugs (Table III) (Figure 1).

$54.3 \%$ of the responders inform patients about oral health after prescribing drugs (Figure 2). However, for those pediatricians who practice at a private hospital, the rate of informing patients about oral health after prescribing drugs was significantly higher (Z:-1.981) and at public hospitals is lower than the university hospitals and others $(p<0.05)$ ( $Z: 3.451)$. This rate was high for associate professors and professors (Z:-3.319) and low for research assistants. $(p<0.05)(z: 3.574)$. In addition to this, this rate was lower for pediatrists who have been working for less than 3 years ( $Z: 2.034)$ and significantly higher for those physicians who have been working for 9-17 years $(p<0.05)(Z:-2.227)$.

Only $27 \%$ of pediatricians felt knowledgeable about informing parents about oral care after druguse. Pediatricians who have been practicing at a university hospital felt less knowledgeable (Z:-2.733), while pediatricians at public hospitals felt more knowledgeable $(p<0.05)(Z: 2.268)$. This rate was low for research assistants (Z:2.995) and high for associate professors/professors $(p<0.05)$ ( $Z$ :-4.393). Also, this rate was significantly lower for pediatricians who have been working less than 3 years (Z:2.497) and significantly higher for those pediatricians who have been working for more than 17 years $(p<0.05)(Z:-2.724)$. Moreover, this rate was lower for those pediatricians caring for 46 or more patients per day $(Z: 2.446)(p<0.05)$.

Additionally, only $17.7 \%$ of responders agreed with the statement that their training about oral health during medical school/pediatrics was sufficient (Table II) (Figure 3). However, pediatricians at a university hospital agreed that they received adequate training $(p<0.05)(Z:-2.078)$. In addition, according to the year-based comparison, although the study period increases, the statement that their training about oral health during medical school/pediatrics was adequately was not rated significantly.

The majority of the pediatricians (68.7\%) recommended that their patients brush their teeth after using a suspension/inhaler drug. 53\% of them recommended

\begin{tabular}{|l|l|}
\hline Table II. Overview of demographic characteristics \\
\hline Demographics & Mean (95\% Cl) \\
\hline Age & $38.88(24-65)$ \\
\hline Years of practice & $11(1-37)$ \\
\hline Daily number of patient & $35.49(3-150)$ \\
\hline & Percentage (\%) \\
\hline Type of practice & \\
\hline University & 18.3 \\
\hline Public hospital & 28.7 \\
\hline Private hospital & 50 \\
\hline Other & 3 \\
\hline Status type & \\
\hline Research asisstant & 23.7 \\
\hline Specialist & 59.3 \\
\hline Assistant professor & 8 \\
\hline Associate professor/Professor & 9 \\
\hline Cl: Confidence interval & \\
\hline &
\end{tabular}


brushing immediately after taking suspension/inhaler drugs. This immediately brushing recommendation rate was low for pediatricians at public hospitals (Z:-3.589) and high for pediatricians at private hospitals $(p<0.05)(Z: 2.503)$. This rate was high for research assistants (Z:-3.285) and low for associate professors/professors $(p<0.05)(Z: 3.291)$. However only $34.7 \%$ of responders advised brushing teeth 20 minutes after taking these types of drugs (Figure 3).

We asked the pediatricians the names of the most common analgesics, antibiotics, inhalers and iron preparations that they frequently prescribe. With respect to iron preparations, pediatricians prescribed Ferro Sanol (31\%), Ferrum (27.7\%) as the most common drug, and Sidefer $(10.7 \%)$ as the second most common drug; with respect to antibiotics, they prescribed Augmentin (47\%), Klamoks (23\%) as the most common drug, and Macrol (10 \%) as the second most common drug; with respect to analgesics, they prescribed Calpol (54.7\%), Parol (19.3\%) as the most common drug and Dolven $(25.7 \%)$ as the second most common; with respect to inhaler preparations, they prescribed Ventolin (68\%), Flixotide (15.3\%) as the most common drug, and Pulmicort $(13.3 \%)$ as the second most common.

Moreover; it was asked to the pediatricians 'What would be your approach when a parent complains about coloration/ tooth decay/erosion which occurs after medication use?' $42 \%$ of the pediatricians replied that they would try to solve

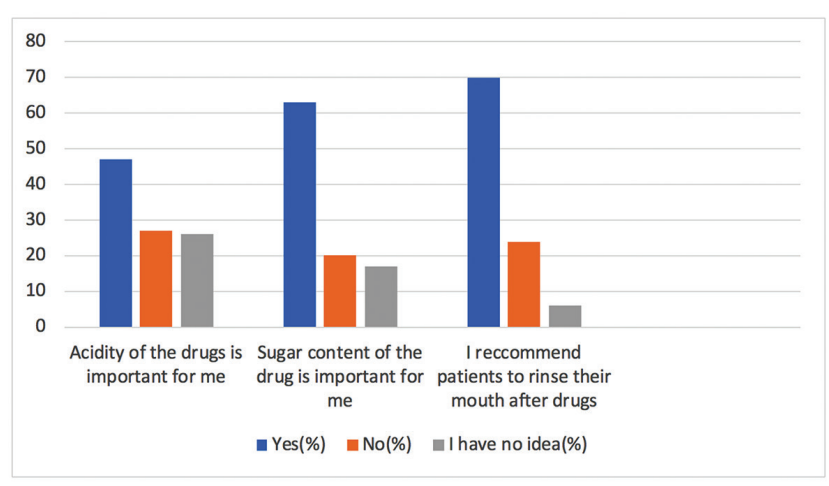

Figure 1. Attitudes of pediatricians the problem by giving advice or take action such as change of medication, discontinuation of medication, better dental care and mouth rinsing. 57.5\% of them answered that when they encountered such a problem, they would consult with a dentist or pediatric dentist. $0.5 \%$ of them answered that this problem is expected as a possible side effect and that they would state to the patient that it was normal. Especially, tooth staining was considered normal and there was no need to take any action.

The total recommendation scores of those pediatricians practicing at private hospitals are significantly higher compared to those working in public hospitals, university hospitals or others $(p<0.05)$.

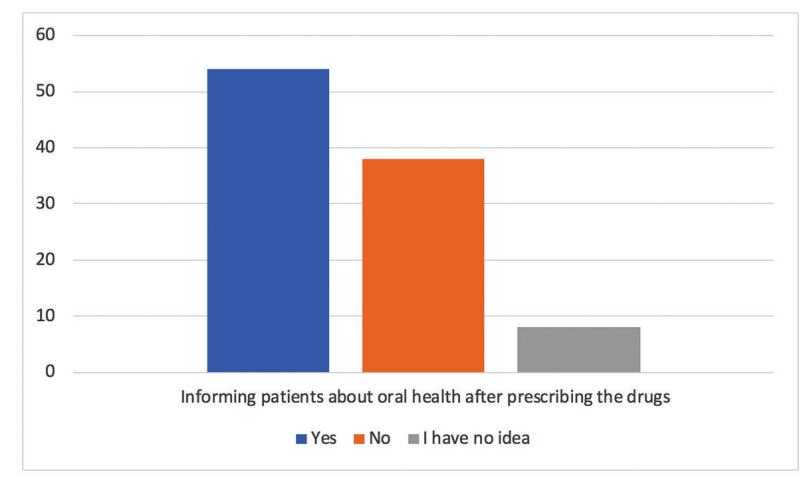

Figure 2. Informing patients about oral heath

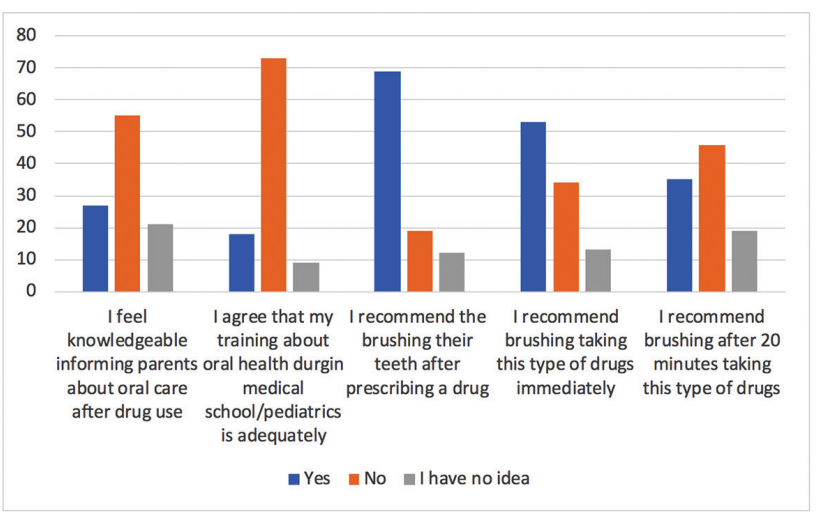

Figure 3. Practices of pediatricians

Table III. Attitudes and practices of the responders

\begin{tabular}{|l|l|l|l|}
\hline & Agree (\%) & Disagree (\%) & Not sure (\%) \\
\hline The acidity of preparation I've prescribed is important for me. & $141(47)$ & $81(27)$ & $78(26)$ \\
\hline The sugar content of preparation I've prescribed is important for me. & $188(62.7)$ & $61(20.3)$ & $51(17)$ \\
\hline I reccommend my patients rinse their mouth after prescribing suspension/inhaler drug. & $211(70.3)$ & $71(23.7)$ & $18(6)$ \\
\hline I inform my patients about oral health after prescribing drug. & $163(54.3)$ & $113(37.7)$ & $24(8)$ \\
\hline I feel knowledgeable about informing parents oral heathcare after medication use. & $81(27)$ & $165(55)$ & $54(18)$ \\
\hline I think my training about oral health during medical school/pediatrics is adequately. & $53(17.7)$ & $219(73)$ & $28(9.3)$ \\
\hline
\end{tabular}




\section{Discussion}

This is the first study to analyze data from a sample of pediatricians to assess their knowledge, attitude and practices concerning the effects of medication use on oral health.

Subjects related with oral health are included in only $33 \%$ of medical schools in many European countries' pediatrics training programmes (11). In contrast, in a US study, the vast majority of pediatricians received oral hygiene education in medical school (12). A high percentage of responders $(73 \%)$ in the present study reported that their education about oral health during medical school/pediatrics was insufficient. When evaluating the findings of this study, we believe that the awareness of pediatricians about oral health should be improved. Inadequate training about oral health can be a challenge for pediatricians in the providing for children's oral health.

Most of the responders (90\%) knew about the tooth staining effect of iron preparations, however, most of them were not aware of the relationship between pediatric suspension forms of drugs and caries formation (42\%) or dental erosion (50.7\%). Also, most of them were not aware of the relationship between inhaler drugs and caries formation (59\%) or dental erosion (44.7\%).

The European Academy of Pediatric Dentistry (EAPD) recommends no intake of sugar containing drinks or sweetened baby bottles especially at nighttime. This recommendation is based on 'common sense' due to the etiologic role of sugar in early childhood caries (13). Although there is no part about drugs in the recommendations, care should be taken not to take some drugs at night because of their content. $76.3 \%$ of the responders in the present study agreed that taking drugs at nighttime may increase the risk of dental caries.

After taking drugs, immediate toothbrushing is not recommended. It should be delayed for at least 20 minutes after an erosive attack and possibly up to 60 minutes because of the increased risk of abrasive wear on the softened/eroded surface (9). This study clearly indicates that the participants are not aware of this recommendation since only $34.7 \%$ of them advised brushing teeth 20 minutes after taking these types of drugs. 53\% of them recommended brushing immediately after taking suspension/inhaler drugs. The recommendation rate for immediate brushing was lower for associate professors/professors $(p<0.05)$. This result increases concerns about the quality of the educational content in medical schools concerning oral health and it is seen that awareness has been created through practical and academic experience.
Some pediatric drugs are highly recommended by the participants in the present study, the oral and dental effects of them are unknown so further studies are necessary with respect to the dental and oral effects of these drugs.

Only $27 \%$ of the study population feel knowledgeable about informing parents about oral healthcare after medication use, which is lower compared to a previous study (12) However, the rate for those pediatricians with more years of working experience was higher. In the study by Adamos et al. (11), it was seen that half (55\%) of pediatricians with more than 10 years in practice felt confident about oral health in children, compared to $36 \%$ of the professionals with 5-10 years in practice and $31 \%$ of those with less than 5 years in practice $(p<0.05)$. While the majority of the pediatricians did not feel sufficient, the rate of informing patients concerning oral health issues increased with the pediatricians level of experience. This indicates an ongoing lack of medical education related to oral health for pediatricians.

This study demonstrates that with respect to the rate of informing patients, practitioners at private hospital were higher. Also, associate professors/professors' rate was higher than research assistants. This rate of informing patients can be associated with the number of intensive patients.

$54.3 \%$ of responders informed patients about oral health after prescribing drugs. The rate in private hospitals was higher. This rate is high for associate professors and professors and low for research assistants. In addition to this, the rate was lower for those pediatrists who had been working for less than 3 years and significantly higher for those physicians who had been working for 9-17 years. According to these results, an awareness about the effects of drugs on oral health develops through practical experience.

\section{Conclusions}

The results of this study provide information about need for oral health education programmes for pediatricians. Although most practitioners often encounter oral and dental effects of the drugs they recommend, they do not feel sufficiently knowledgeable to inform patients. There is a need to organize the curriculum related to oral health in pediatrics education and to increase the level of knowledge of pediatricians through educational programmes.

\section{Ethics}

Ethics Committee Approval: Ethics approval was obtained from the institutional ethics and research 
committee of the University of Health Sciences (12/18) (approval number: 18/103).

Informed Consent: Informed consent was taken from the people who participated in study.

Peer-review: Externally peer-reviewed.

\section{Authorship Contributions}

Concept: Ş.T.K., Design: Ş.T.K., Data Collection or Processing: G.G.P., C.A., Analysis or Interpretation: E.A.K., Literature Search: Ş.T.K., Writing: Ş.T.K.

Conflict of Interest: No conflict of interest was declared by the authors.

Financial Disclosure: The authors declared that this study received no financial support.

\section{References}

1. Maguire A, Baqir W, Nunn JH. Are sugars-free medicines more erosive than sugars-containing medicines? An in vitro study of paediatric medicines with prolonged oral clearance used regularly and long-term by children. Int I Paediatr Dent 2007; 17:231-8.

2. Kenny DJ, Somaya P. Sugar load of oral liquid medications on chronically ill children. J Can Dent Assoc 1989;55:43-6.

3. Duward C, Thou T. Dental caries and sugar containing liquid medicines for children in New Zealand NZ Dent I 1997;93:124-9.

4. Bigeard L. The role of medication and sugars in pediatric dental patients. Dent Clin North Am 2000; 44:443-56.
5. Kumar A, Kumar V, Singh J, Hooda A, Dutta S. Drug-induced discoloration of teeth: an updated review. Clin Pediatr (Phila) 2012; 51:181-5.

6. Addy M, Moran J. Mechanisms of stain formation on teeth, in particular associated with metal ions and antiseptics. Adv Dent Res 1995; 9:450-6.

7. Costa CC, Almeida ICS, Costa Filho LC. Erosive effect of an antihistamine-containing syrup on primary enamel and its reduction by fluoride dentifrice. Int J Paediatr Dent 2006; 16:174180.

8. Kato MT, Sale-Peres SH, Buzalaf MA. Effect of iron on acid demineralisation of bovine enamel blocks by a soft drink. Arch Oral Biol 2007; 52:1109-11.

9. Attin T, Knofel S, Buchalia W, Tutuncu R. In situ evaluation of different remineralisation periods to decrease brushing abrasion of demineralised enamel. Caries Res 2001; 35:216-22.

10. Krol DM. Educating pediatricians on children's oral health: past, present and future. Pediatrics 2004; 13:487-93.

11. Adamos H, Zachi G, Stefano del TK, Michailidou, Diego VE, Rita C. Oral health training, knowledge, attitudes and practices of primary care paediatricians: a European survey. European Journal of Pediatrics 2018; 177:675-81.

12. Lewis CW, Grossman DC, Domoto PK, Deyo RA. The role of the pediatrician in the oral health of children: a national survey. Pediatrics 2000; 106:84.

13. Tinanoff N, Palmer CA. Dietary determinants of dental caries and dietary recommendations for preschool children. I Public Health Dent 2000; 60:197-206. 